\section{Kontrazeption für den Mann}

\section{Durch die Injektion der Kombination aus einem Testosteron und einem Gestagen lässt sich die Spermienbildung wirksam unterdrücken.}

$\mathrm{D}$ ie Gestagen-Testosteron-Kombination wurde in einer prospektiven Studie mit 320 gesunden Männern zwischen 18 und 45 Jahren untersucht, die in einer stabilen Partnerschaft lebten. Alle acht Wochen wurde den Männern insgesamt 26 Wochen lang - eine Kombination aus $200 \mathrm{mg}$ NETE und 1.000 $\mathrm{mg}$ des Testosterons in den Musculus gluteus injiziert. In der anschließenden Wirksamkeitsphase wurden die Injektionen bis zu 56 Wochen lang fortgesetzt. In dieser Zeit hatten die Paare ansonsten ungeschützten Geschlechtsverkehr. Ab der achten Woche nach der letzten Injektion setzte die bis zu einem Jahr dauernde Erholungsphase ein.

In die Wirksamkeitsphase traten Studienteilnehmer ein, wenn die Zahl der Spermien maximal 1 Million/ml betrug.

\title{
Urinkultur vor Operation nur in ausgewählten Fällen?
}

\section{Die Ergebnisse einer italienischen Studie sprechen eher dagegen, vor einem urologischen Eingriff routinemäßig eine Urinkultur anzufertigen, sofern die Patienten eine antibiotische Prophylaxe erhalten.}

G emäß der Leitlinie der European Association of Urology (EAU) stellt eine Bakteriurie bei einem bevorstehenden urologischen Eingriff ein erhebliches Komplikationsrisiko dar, weshalb bei asymptomatischen Patienten eine antibiotische Prophylaxe empfohlen wird. Inwieweit präoperativ ein Nachweis der bakteriellen Pathogene mittels Urinkultur sinnvoll ist, ist umstritten. Dass sich die präoperative Urinanalyse nur in bestimmten Fällen lohnt, legt eine Studie von Cai et al. mit 2.201 Patienten nahe, die vor ihrer urologischen Operation eine EAU-leitliniengerechte Antibiotikaprophylaxe erhielten.

Als asymptomatische Bakteriurie wurde das Vorhandensein von mindestens $105 \mathrm{CFU} / \mathrm{ml}$ definiert; bei den Erregern
Nur vier von insgesamt 266 Partnerinnen wurden schwanger (1,57 pro $100 \mathrm{An}$ wendern), und zwar alle bis zur 16 . Woche in der Wirksamkeitsphase der Studie. Das entspricht den Studienleitern zufolge einem Pearl-Index von 2,18 Schwangerschaften pro 100 Personenjahre. Bei der Anwendung von Kondomen liegt der Index zwischen 2 und 12, bei der Pille zwischen 0,1 und 0,9. Bei keinem der vier Männer kam es zur Azoospermie. Die Zahl der Spermien zum vermuteten Konzeptionszeitpunkt lag bei drei Teilnehmern bis maximal 1 Million $/ \mathrm{ml}$. Beim vierten Mann lag sie wenige Tage vor der Konzeption bei 0,2 und wenige Tage danach bei 1,6 Million/ml. Nach der Erholungsphase von 52 Wochen hatte sich bei 94,8 \% der Männer die Spermienzahl wieder normalisiert.
Wegen unerwünschter Wirkungen wie Akne, Stimmungsschwankungen und Schmerzen an der Einstichstelle wurde die Studie vorzeitig beendet. Die Studienteilnehmer berichteten über insgesamt fast 1.500 unerwünschte Ereignisse. $29 \%$ wurden als sicher mit der Hormonbehandlung assoziiert.

Fazit: Zwei unabhängige Ausschüsse kamen zu unterschiedlichen Ergebnissen bei der Bewertung der Sicherheit dieser Methode zur Kontrazeption, mit der Folge, dass die intramuskulären Injektionen gestoppt wurden. Eine endgültige Antwort darauf, ob tatsächlich die Risiken der Methode, wie sie in der Studie angewendet worden war, den Nutzen überwiegen, könnten die Ergebnisse der Studie jedoch nicht liefern, so die Ärzte.

Peter Leiner

Behre HM et al. Efficacy and Safety of an Injectable Combination Hormonal Contraceptive for Men. J Clin Endocrinol Metab. 2016;doi:10.1210/ jc.2016-2141.

betalaktamase)-produzierende Stämme nachgewiesen. Von diesen hatte lediglich einer eine symptomatische postoperative HWI entwickelt. Der präoperative Nachweis einer $A B$ war nicht mit einem höheren Risiko einer symptomatischen HWI nach dem Eingriff assoziiert. Dagegen bestand ein direkter Zusammenhang mit dem Charlson Comorbidity Index (> 1).

musste es sich um uropathogene Stämme handeln. Die Forscher bildeten zwei Gruppen: Bei den 668 Patienten der Gruppe A war eine asymptomatische Bakteriurie (AB) nachgewiesen, bei Gruppe B (1.533 Patienten) nicht. Eine mikrobiologisch nachgewiesene postoperative Harnwegsinfektion (HWI) trat in insgesamt 8,9\% der Fälle auf. Von den 198 Betroffenen hatten nur 18 Fieber. Ein Drittel davon entwickelte eine Urosepsis.

Die Raten der Patienten mit symptomatischer postoperativer HWI in beiden Gruppen waren annähernd gleich (10,4\% bzw. $8,3 \%)$. Das galt auch für die Urosepsis (0,30 \% bzw. 0,26 \%). In Gruppe A hatte man bei zwölf Patienten Resistenzen gegenüber Fluorchinolone und/oder ESBL(„extended-spectrum-
Fazit: Das präoperative AB-Screening steht bei leitliniengerecht mit antibiotischer Prophylaxe versorgten Patienten in keinem Zusammenhang mit einem erhöhten Risiko einer symptomatischen HWI. Laut Cai et al. lege dies nahe, dass „die routinemäßige präoperative Urinanalyse möglicherweise nur für ausgewählte Patientengruppen " $\mathrm{zu}$ fordern sei, beispielsweise solche mit symptomatischer HWI oder höheren Komorbiditätsscores.

Dr. Elke Oberhofer

Cai T et al. Is Pre-Operative Assessment and Treatment of Asymptomatic Bacteriuria Necessary for Reducing the Risk of Post-Operative Symptomatic Urinary Tract Infections after Urological Surgical Procedures? Urology. 2016, doi:10.1016/j.urology.2016.10.016. 